\title{
Bilarge Neutrino Mixing and Abelian Flavor Symmetry
}

\author{
Gui-Jun Ding ${ }^{a}$, S. Morisi ${ }^{b, c}$, and J. W. F. Valle ${ }^{b}$ \\ ${ }^{a}$ Department of Modern Physics, \\ University of Science and Technology of China, Hefei, Anhui 230026, China \\ ${ }^{b}$ AHEP Group, Instituto de Física Corpuscular - C.S.I.C./Universitat de València \\ Edificio de Institutos de Paterna, Apartado 22085, E-46071 València, Spain \\ ${ }^{c}$ Institut für Theoretische Physik und Astrophysik, \\ Universität Würzburg, 97074 Würzburg, Germany
}

\begin{abstract}
We explore two bilarge Neutrino Mixing anzätze within the context of Abelian flavor symmetry theories: $\left(\mathrm{BL}_{1}\right) \sin \theta_{12} \sim \lambda, \sin \theta_{13} \sim \lambda, \sin \theta_{23} \sim \lambda$, and $\left(\mathrm{BL}_{2}\right) \sin \theta_{12} \sim \lambda$, $\sin \theta_{13} \sim \lambda, \sin \theta_{23} \sim 1-\lambda$. The first pattern is proposed by two of us and is favored if the atmospheric mixing angle $\theta_{23}$ lies in the first octant, while the second one is preferred for the second octant of $\theta_{23}$. In order to reproduce the second texture, we find that the flavor symmetry should be $U(1) \times Z_{m}$, while for the first pattern the flavor symmetry should be extended to $U(1) \times Z_{m} \times Z_{n}$ with $m$ and $n$ of different parity. Explicit models for both mixing patterns are constructed based on the flavor symmetries $U(1) \times Z_{3} \times Z_{4}$ and $U(1) \times Z_{2}$. The models are extended to the quark sector within the framework of $S U(5)$ grand unified theory in order to give a successful description of quark and lepton masses and mixing simultaneously. Phenomenological implications are discussed.
\end{abstract}




\section{Introduction}

Our knowledge of the neutrino oscillation parameters has enormously improved in recent years. In particular the Daya Bay [1], RENO [2] and Double Chooz [3] Collaborations have established that the reactor mixing angle $\theta_{13}>0$ at about $5 \sigma$ confidence level, confirming the early hints for a nonzero $\theta_{13}$ [4, 5]. Recent global analyses [6, 7] of neutrino oscillation parameters, including the data released at the Neutrino-2012 conference, find that $\theta_{13}$ is nonzero at about $10 \sigma$, and non-maximal atmospheric mixing angle $\theta_{23}$ is preferred. However, it still isn't clear which octant $\theta_{23}$ lies in. The global fit of Ref. [6], prefers $\theta_{23}$ in the second octant with the best fit value $\sin ^{2} \theta_{23}=0.613$ (0.600) for normal (inverted) neutrino mass hierarchy, although this hint is quite marginal and first octant values of $\theta_{23}$ are well inside the $1 \sigma$ range for normal hierarchy and at $1.2 \sigma$ for inverted spectrum. While the independent phenomenological analyses of atmospheric neutrino data in Ref. [7] obtain a preference for $\theta_{23}$ in the first octant for both mass hierarchies and exclude maximal mixing at the $2 \sigma$ level, the best fit value is found to be $\sin ^{2} \theta_{23}=0.386$ (0.392) for normal (inverted) neutrino spectrum. Alternative recent global fits claim both the first and second $\theta_{23}$ octants are possible 8]. As for the mass-squared difference, the best fit values of $\Delta m_{\text {sol }}^{2}$ and $\Delta m_{\text {atm }}^{2}$ are $7.62 \times 10^{-5} \mathrm{eV}^{2}$ and $2.55(2.43) \times 10^{-3} \mathrm{eV}^{2}$ respectively, which lead to $\Delta m_{\mathrm{sol}}^{2} / \Delta m_{\mathrm{atm}}^{2} \simeq 0.030(0.031)$. Here the values shown in parentheses correspond to the inverted neutrino mass hierarchy. Note that the three groups give almost the same $3 \sigma$ ranges for the lepton mixing parameters.

From the theoretical or model-building point of view, one implication of this significant experimental progress is that it excludes the tri-bimaximal mixing ansatz for neutrino mixing [9], unless the underlying theory is capable of providing sufficiently large corrections. So far many suggestions have been advanced to explain the new data, in particular the largish $\theta_{13}[10-15]$. Instead of seeking for new mass-independent lepton mixing matrices to replace the tri-bimaximal pattern [11 13, which may be derived from certain discrete flavor symmetries, Ref. 14 proposed a novel Wolfenstein-like ansatz for the neutrino mixing matrix. In this scheme, all three lepton mixing angles are assumed to be of the same order to first approximation

$$
\sin \theta_{12} \sim \lambda, \quad \sin \theta_{13} \sim \lambda, \quad \sin \theta_{23} \sim \lambda,
$$

where $\lambda \simeq 0.23$ is the Cabibbo angle, and the symbol " $\sim$ " implies that the above relations contain unknown factors of order one, the freedom in these factors can be used to obtain an adequate description of the neutrino mixing. Inspecting the global data fitting [6] 8], we see that $\sin \theta_{12} \simeq 2.5 \lambda$ and $\sin \theta_{13} \simeq \lambda / \sqrt{2}$, which is proposed in the so-called Tri-bimaximalCabibbo mixing [15] and also appeared in the context of quark-lepton complementarity [16]. Such bilarge mixing pattern [14] would clearly provide a good leading order approximation for the current neutrino mixing pattern, if the atmospheric neutrino mixing angle $\theta_{23}$ turns out to lie in the first octant. However, the second octant of $\theta_{23}$ can not be ruled out and is supported by the analyses in Refs. 6, 8. In this case the texture

$$
\sin \theta_{12} \sim \lambda, \quad \sin \theta_{13} \sim \lambda, \quad \sin \theta_{23} \sim 1-\lambda
$$


could be taken as a viable model-building standard. We shall refer to two mixing patterns as $\mathrm{BL}_{1}$ and $\mathrm{BL}_{2}$ textures respectively. The difference between $\mathrm{BL}_{1}$ and $\mathrm{BL}_{2}$ mixing lies in the order of magnitude of the atmospheric mixing angle $\theta_{23}$; the $\mathrm{BL}_{1}$ mixing pattern would be favored if future experiments establish that $\theta_{23}$ belongs to the first octant and the deviation from maximal mixing is somewhat large; otherwise, $\mathrm{BL}_{2}$ mixing is preferred. It is well-known that the observed hierarchies of masses and flavor mixing in the quarks and charged leptons sectors can be conveniently characterized by the Cabibbo angle. As a result the $\mathrm{BL}_{1}$ and $\mathrm{BL}_{2}$ parametrization may have deep implications for the theoretical formulation of the ultimate unified theory of flavor. A lot of work in the literature has demonstrated that the smallness and hierarchy of the quark masses and mixing angles can be naturally generated in theories which, at low energy, are described effectively by an Abelian horizontal symmetry, which is explicitly broken by a small parameter [17 19]. It certainly follows a natural path to try and apply these ideas on Abelian family symmetries developed for the quarks to the lepton sector. In this work, we shall investigate whether and how the $\mathrm{BL}_{1}$ and $\mathrm{BL}_{2}$ textures can be reproduced naturally from the Abelian horizontal flavor symmetry. For generality we assume that the light neutrino masses arise from lepton-number-violating effective Weinberglike operators.

The paper is organized as follows. In section 2, we present the effective low energy theory for the Abelian $U(1)$ flavor symmetry and its extension to $U(1) \times Z_{m} \times Z_{n}$. We find that, in order to produce the $\mathrm{BL}_{1}$ texture without fine-tuning, the family symmetry should be $U(1) \times Z_{m} \times Z_{n}$ with $m$ and $n$ of opposite parity. Models for the $\mathrm{BL}_{1}$ and $\mathrm{BL}_{2}$ schemes are constructed in section 3 and section 4 respectively. These models are extended to include quarks within the $S U(5)$ grand unified theory (GUT), the observed patterns of both quark and lepton masses and flavor mixings are reproduced, and the general phenomenological predictions of the models are discussed. Finally, our conclusions are summarized in section 5.

\section{Theoretical Framework}

Our theoretical framework is defined as follows. For definiteness we consider a low energy effective theory with the same particle content as the supersymmetric Standard Model (SM). In addition to supersymmetry and the SM gauge symmetry, we introduce a horizontal $U(1)$ symmetry and a SM singlet chiral superfield $\Theta$ which is charged under the $U(1)$ family symmetry; without loss of generality, we normalize its charge to -1 . The effective Yukawa couplings of the quarks and leptons are generated from nonrenormalizable superpotential terms of the form

$$
\begin{aligned}
W & =\left(y_{u}\right)_{i j} Q_{i} U_{j}^{c} H_{u}\left(\frac{\Theta}{\Lambda}\right)^{F\left(Q_{i}\right)+F\left(U_{j}^{c}\right)}+\left(y_{d}\right)_{i j} Q_{i} D_{j}^{c} H_{d}\left(\frac{\Theta}{\Lambda}\right)^{F\left(Q_{i}\right)+F\left(D_{j}^{c}\right)} \\
& +\left(y_{e}\right)_{i j} L_{i} E_{j}^{c} H_{d}\left(\frac{\Theta}{\Lambda}\right)^{F\left(L_{i}\right)+F\left(E_{j}^{c}\right)}+\left(y_{\nu}\right)_{i j} \frac{1}{\Lambda} L_{i} L_{j} H_{u} H_{u}\left(\frac{\Theta}{\Lambda}\right)^{F\left(L_{i}\right)+F\left(L_{j}\right)}
\end{aligned}
$$


where $H_{u, d}$ are Higgs doublets, $Q_{i}$ and $L_{i}$ are the left-handed quark and lepton doublets respectively, $U_{j}^{c}, D_{j}^{c}$ and $E_{j}^{c}$ are the right-handed up-type quark, down-type quark and charged lepton superfields respectively, and $i, j$ are generation indices. The parameter $\Lambda$ is the cutoff scale of the $U(1)$ symmetry, and $F(\psi)$ denotes the $U(1)$ charge of the field $\psi$. Note that $F\left(H_{u}\right)$ and $F\left(H_{d}\right)$ do not appear in the exponents since one can always set the horizontal charges of the Higgs doublet $H_{u}$ and $H_{u}$ to zero by redefinition of the $U(1)$ charges. The last term of Eq.(3) is the high-dimensional version of the effective lepton-number-violating Weinberg operator.

For the Froggatt-Nielsen flavon field $\Theta$, the supersymmetric action contains a FayetIliopoulos term and the associated D-term in the scalar potential provides a large vacuum expectation value $(\mathrm{VEV})$ for the scalar component of $\Theta$. The D-term in the potential is given by

$$
V_{D}=\frac{1}{2}\left(M_{F I}^{2}-g_{\Theta}|\Theta|^{2}\right)^{2}
$$

where $M_{F I}^{2}$ is the Fayet-Iliopoulos term. The vanishing of $V_{D}$ requires

$$
|\langle\Theta\rangle|=M_{F I} / \sqrt{g_{\Theta}}
$$

We note that this flavor symmetry breaking mechanism is also frequently exploited in discrete flavor symmetry model building [20]. Once the horizontal symmetry is broken by the $\operatorname{VEV}\langle\Theta\rangle$, one obtains the quark and lepton mass matrices whose elements are suppressed by powers of the small parameter $\langle\Theta\rangle / \Lambda$, which for simplicity is usually assumed to be characterized by the Cabibbo angle, i.e., $\lambda=\langle\Theta\rangle / \Lambda$, then we have

$$
\begin{array}{ll}
\left(M_{u}\right)_{i j}=\left(y_{u}\right)_{i j} \lambda^{F\left(Q_{i}\right)+F\left(U_{j}^{c}\right)} v_{u}, & \left(M_{d}\right)_{i j}=\left(y_{d}\right)_{i j} \lambda^{F\left(Q_{i}\right)+F\left(D_{j}^{c}\right)} v_{d}, \\
\left(M_{e}\right)_{i j}=\left(y_{e}\right)_{i j} \lambda^{F\left(L_{i}\right)+F\left(E_{j}^{c}\right)} v_{u}, & \left(M_{\nu}\right)_{i j}=\left(y_{\nu}\right)_{i j} \lambda^{F\left(L_{i}\right)+F\left(L_{j}\right)} \frac{v_{u}^{2}}{\Lambda}
\end{array}
$$

where $v_{u, d}=\left\langle H_{u, d}\right\rangle$ is the electroweak scale VEV of the Higgs doublet $H_{u, d}$. The factors $\left(y_{u}\right)_{i j},\left(y_{d}\right)_{i j},\left(y_{e}\right)_{i j}$ and $\left(y_{\nu}\right)_{i j}$ are not constrained by the flavor symmetry and are usually assumed to be of order one, the freedom in these factors is used in order to obtain a quantitative description of the fermion masses and flavor mixings. Since the holomorphicity of the superpotential forbids nonrenormalizable terms with a negative power of the superfield $\Theta$, one has $\left(M_{u}\right)_{i j}=0$ if $F\left(Q_{i}\right)+F\left(U_{j}^{c}\right)<0$. Similarly $\left(M_{d}\right)_{i j}=0$ if $F\left(Q_{i}\right)+F\left(D_{j}^{c}\right)<0$, $\left(M_{e}\right)_{i j}=0$ if $F\left(L_{i}\right)+F\left(E_{j}^{c}\right)<0$, and $\left(M_{\nu}\right)_{i j}=0$ if $F\left(L_{i}\right)+F\left(L_{j}\right)<0$.

In our framework, the light neutrino masses are generated by the high-dimensional effective Weinberg operators shown in the last term of Eq.(3), consequently, the light neutrinos are Majorana particles and its mass matrix $M_{\nu}$ is symmetric with $\left(M_{\nu}\right)_{i j}=\left(M_{\nu}\right)_{j i}$. Furthermore, if all the horizontal charges are positive, the hierarchial structure of the mass

\footnotetext{
${ }^{1}$ Note that if we introduce three right-handed neutrino superfields $N_{i}^{c}$ to generate light neutrino mass via type I seesaw mechanism, the structure of the light neutrino mass matrix $M_{\nu}$ is independent of the $N_{i}^{c}$ charge assignments [24, 25, unless there are holomorphic zeros in neutrino Dirac mass matrix $M_{D}$ or in Majorana mass matrix $M_{N}$ for the heavy fields $N^{c}$.
} 
matrices shown in Eq.([6]) allows a simple order of magnitude estimate for the various mass ratios and mixing angles:

$$
\begin{aligned}
& \frac{m_{u_{i}}}{m_{u_{j}}} \sim \lambda^{F\left(Q_{i}\right)-F\left(Q_{j}\right)+F\left(U_{i}^{c}\right)-F\left(U_{j}^{c}\right)}, \quad \frac{m_{d_{i}}}{m_{d_{j}}} \sim \lambda^{F\left(Q_{i}\right)-F\left(Q_{j}\right)+F\left(D_{i}^{c}\right)-F\left(D_{j}^{c}\right)}, \quad V_{i j} \sim \lambda^{F\left(Q_{i}\right)-F\left(Q_{j}\right)}, \\
& \frac{m_{i}}{m_{j}} \sim \lambda^{2\left[F\left(L_{i}\right)-F\left(L_{j}\right)\right]}, \quad \frac{m_{\ell_{i}}}{m_{\ell_{j}}} \sim \lambda^{F\left(L_{i}\right)-F\left(L_{j}\right)+F\left(E_{i}^{c}\right)-F\left(E_{j}^{c}\right)}, \quad \sin \theta_{i j} \sim \lambda^{F\left(L_{i}\right)-F\left(L_{j}\right)} \quad(7)
\end{aligned}
$$

where $m_{i}$ is the light neutrino mass, and $V_{i j}$ denotes the element of the quark CabibboKobayashi-Maskawa quark-mixing matrix (CKM) mixing matrix. We note that the sign " " implies that there is an unknown order one coefficient in each relation, so that the actual value of the mass ratios and mixing angles may slightly depart from the naive "power counting" estimate. Moreover, if some fields carry negative $F$ charges, then holomorphy plays an important role and the estimates (7) could be violated as well. For the $\mathrm{BL}_{1}$ mixing pattern, both $\sin \theta_{12}$ and $\sin \theta_{23}$ are of order $\lambda$, then we should require

$$
F\left(L_{1}\right)=F\left(L_{2}\right)+1, \quad F\left(L_{2}\right)=F\left(L_{3}\right)+1
$$

This implies $F\left(L_{1}\right)=F\left(L_{3}\right)+2$, as a result, we have $\sin \theta_{13} \sim \lambda^{2}$. Therefore we conclude that the $\mathrm{BL}_{1}$ mixing pattern can not be naturally produced from a pure $U(1)$ flavor symmetry. Turning to the $\mathrm{BL}_{2}$ mixing pattern given by $\sin \theta_{23} \sim 1, \sin \theta_{12} \sim \lambda$ and $\sin \theta_{13} \sim \lambda$, one should choose

$$
F\left(L_{2}\right)=F\left(L_{3}\right)=F\left(L_{1}\right)-1
$$

Then we have the $(2 i)$ and $(3 i)(i=1,2,3)$ entries of the charged lepton mass matrix are of the same order, hence the diagonalization of the charged lepton mass matrix leads to large 2-3 mixing. In addition, we obtain

$$
M_{\nu} \sim \lambda^{2 F\left(L_{3}\right)}\left(\begin{array}{ccc}
\lambda^{2} & \lambda & \lambda \\
\lambda & 1 & 1 \\
\lambda & 1 & 1
\end{array}\right) \frac{v_{u}^{2}}{\Lambda}
$$

Clearly the (2-3) sector of the light neutrino mass matrix has a democratic structure, thus large mixing in this (2-3) sector is naturally obtained. However, barring the presence of special cancellations, the masses of the second and the third light neutrinos are typically expected to be of the same order in this case. As a result, the three light neutrinos are quasi-degenerate and strong parameter fine-tuning is required in order to account for the hierarchy between the measured mass squared differences $\Delta m_{\mathrm{sol}}^{2}$ and $\Delta m_{\mathrm{atm}}^{2}$.

In order to avoid this kind of fine-tuning in obtaining an acceptable pattern of neutrino oscillation parameters, we must go beyond the pure $U(1)$ flavor symmetry case considered above. Let us now move to the extended flavor symmetry $U(1) \times Z_{m} \times Z_{n} \subset U(1) \times$ 
$U(1)^{\prime} \times U(1)^{\prime \prime}$. This kind of Abelian symmetry is somewhat complex and not yet fully discussed, as far as we know, since most of the previous work concentrated on $U(1)$ or $U(1) \times Z_{m} \subset U(1) \times U(1)^{\prime}$ flavor symmetry. We now consider [18, 25] three SM singlet superfields $\Theta_{1}, \Theta_{2}$ and $\Theta_{3}$ with the horizontal charges

$$
\Theta_{1}:(-1,0,0), \quad \Theta_{2}:(0,-1,0), \quad \Theta_{3}:(0,0,-1)
$$

In exactly the same way as the single $U(1)$ case, the three flavons $\Theta_{1}, \Theta_{2}$ and $\Theta_{3}$ could get non-vanishing VEVs determined by corresponding the D-terms. In general the $\operatorname{VEVs}\left\langle\Theta_{1}\right\rangle$, $\left\langle\Theta_{2}\right\rangle$ and $\left\langle\Theta_{3}\right\rangle$ are different [18,25]. For simplicity, we take in what follows: $\left\langle\Theta_{1}\right\rangle / \Lambda \sim \lambda$, $\left\langle\Theta_{2}\right\rangle / \Lambda \sim \lambda$ and $\left\langle\Theta_{3}\right\rangle / \Lambda \sim \lambda$. The effective Yukawa couplings are given by extending Eq.(3) with new flavons $\Theta_{1}, \Theta_{2}$ and $\Theta_{3}$ as follows:

$$
\begin{aligned}
W= & \left(y_{u}\right)_{i j} Q_{i} U_{j}^{c} H_{u}\left(\frac{\Theta_{1}}{\Lambda}\right)^{F\left(Q_{i}\right)+F\left(U_{j}^{c}\right)}\left(\frac{\Theta_{2}}{\Lambda}\right)^{\left[Z_{m}\left(Q_{i}\right)+Z_{m}\left(U_{j}^{c}\right)\right]}\left(\frac{\Theta_{3}}{\Lambda}\right)^{\left[Z_{n}\left(Q_{i}\right)+Z_{n}\left(U_{j}^{c}\right)\right]} \\
& +\left(y_{d}\right)_{i j} Q_{i} D_{j}^{c} H_{d}\left(\frac{\Theta_{1}}{\Lambda}\right)^{F\left(Q_{i}\right)+F\left(D_{j}^{c}\right)}\left(\frac{\Theta_{2}}{\Lambda}\right)^{\left[Z_{m}\left(Q_{i}\right)+Z_{m}\left(D_{j}^{c}\right)\right]}\left(\frac{\Theta_{3}}{\Lambda}\right)^{\left[Z_{n}\left(Q_{i}\right)+Z_{n}\left(D_{j}^{c}\right)\right]} \\
& +\left(y_{e}\right)_{i j} L_{i} E_{j}^{c} H_{d}\left(\frac{\Theta_{1}}{\Lambda}\right)^{F\left(L_{i}\right)+F\left(E_{j}^{c}\right)}\left(\frac{\Theta_{2}}{\Lambda}\right)^{\left[Z_{m}\left(L_{i}\right)+Z_{m}\left(E_{j}^{c}\right)\right]}\left(\frac{\Theta_{3}}{\Lambda}\right)^{\left[Z_{n}\left(L_{i}\right)+Z_{n}\left(E_{j}^{c}\right)\right]} \\
& +\left(y_{\nu}\right)_{i j} \frac{1}{\Lambda} L_{i} L_{j} H_{u} H_{u}\left(\frac{\Theta_{1}}{\Lambda}\right)^{F\left(L_{i}\right)+F\left(L_{j}\right)}\left(\frac{\Theta_{2}}{\Lambda}\right)^{\left[Z_{m}\left(L_{i}\right)+Z_{m}\left(L_{j}\right)\right]}\left(\frac{\Theta_{3}}{\Lambda}\right)^{\left[Z_{n}\left(L_{i}\right)+Z_{n}\left(L_{j}\right)\right]}
\end{aligned}
$$

where $Z_{m, n}(\psi)$ is the $Z_{m, n}$ charge of the field $\psi$, and the brackets [...] around the exponents denote that we are modding out by $m(n)$ according to the $Z_{m}\left(Z_{n}\right)$ addition rule, namely,

$$
\left[Z_{m}\left(Q_{i}\right)+Z_{m}\left(U_{j}^{c}\right)\right]= \begin{cases}r & \text { if } r<m \\ r-m & \text { if } r \geq m\end{cases}
$$

where $r=Z_{m}\left(Q_{i}\right)+Z_{m}\left(U_{j}^{c}\right)$. We note that the charge assignments of the Higgs doublets $H_{u}$ and $H_{d}$ have been set to $(0,0,0)$ by redefining the flavor symmetry charges of the fields. Thus, the fermion mass matrix can be expressed in term of the horizontal charges as

$$
\begin{aligned}
& \left(M_{u}\right)_{i j}=\left(y_{u}\right)_{i j} \lambda^{F\left(Q_{i}\right)+F\left(U_{j}^{c}\right)+\left[Z_{m}\left(Q_{i}\right)+Z_{m}\left(U_{j}^{c}\right)\right]+\left[Z_{n}\left(Q_{i}\right)+Z_{n}\left(U_{j}^{c}\right)\right]} v_{u} \text {, } \\
& \left(M_{d}\right)_{i j}=\left(y_{d}\right)_{i j} \lambda^{F\left(Q_{i}\right)+F\left(D_{j}^{c}\right)+\left[Z_{m}\left(Q_{i}\right)+Z_{m}\left(D_{j}^{c}\right)\right]+\left[Z_{n}\left(Q_{i}\right)+Z_{n}\left(D_{j}^{c}\right)\right]} v_{d} \text {, } \\
& \left(M_{e}\right)_{i j}=\left(y_{e}\right)_{i j} \lambda^{F\left(L_{i}\right)+F\left(E_{j}^{c}\right)+\left[Z_{m}\left(L_{i}\right)+Z_{m}\left(E_{j}^{c}\right)\right]+\left[Z_{n}\left(L_{i}\right)+Z_{n}\left(E_{j}^{c}\right)\right]} v_{d} \text {, } \\
& \left(M_{\nu}\right)_{i j}=\left(y_{\nu}\right)_{i j} \lambda^{F\left(L_{i}\right)+F\left(L_{j}\right)+\left[Z_{m}\left(L_{i}\right)+Z_{m}\left(L_{j}\right)\right]+\left[Z_{n}\left(L_{i}\right)+Z_{n}\left(L_{j}\right)\right]} \frac{v_{u}^{2}}{\Lambda} .
\end{aligned}
$$

Consider the quark sector, the flavor mixing angles there are given by

$$
\begin{aligned}
& V_{i j}^{u} \sim \lambda^{\left(F\left(Q_{i}\right)+F\left(U_{j}^{c}\right)\right)-\left(F\left(Q_{j}\right)+F\left(U_{j}^{c}\right)\right)+\left[Z_{m(n)}\left(Q_{i}\right)+Z_{m(n)}\left(U_{j}^{c}\right)\right]-\left[Z_{m(n)}\left(Q_{j}\right)+Z_{m(n)}\left(U_{j}^{c}\right)\right],}
\end{aligned}
$$

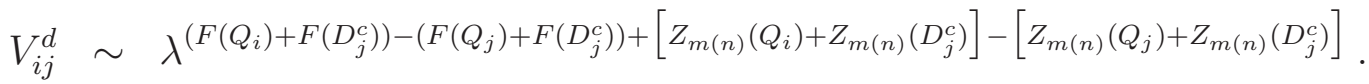

For $m=n=0$ the CKM matrix elements describing the charged current weak interaction of quarks behave approximatively as $V_{i j}^{u, d} \sim \lambda^{F\left(Q_{i}\right)-F\left(Q_{j}\right)}$ and therefore the CKM mixing 
$V_{C K M}=V^{u \dagger} \cdot V^{d}$ is expected to scale as $V_{C K M_{i j}} \sim \lambda^{F\left(Q_{i}\right)-F\left(Q_{j}\right)}$. In order to compare with the pure $U(1)$ horizontal symmetry case, we can define an effective flavor charge in the general case $m \neq n \neq 0$ as

$$
F_{e f f}(\psi)=F(\psi)+Z_{m}(\psi)+Z_{n}(\psi)
$$

Then it is clear that

$$
V_{C K M_{i j}} \sim \lambda^{F_{e f f}\left(Q_{i}\right)-F_{e f f}\left(Q_{j}\right) \pm \alpha m \pm \beta n},
$$

where $\alpha, \beta=0,1$ and we have used Eq. (13) and the fact that

$$
\begin{aligned}
& {\left[Z_{m}\left(Q_{i}\right)+Z_{m}\left(U_{j}^{c}\right)\right]-\left[Z_{m}\left(Q_{j}\right)+Z_{m}\left(U_{j}^{c}\right)\right]=Z_{m}\left(Q_{i}\right)-Z_{m}\left(Q_{j}\right) \pm \alpha m} \\
& {\left[Z_{m}\left(Q_{i}\right)+Z_{m}\left(D_{j}^{c}\right)\right]-\left[Z_{m}\left(Q_{j}\right)+Z_{m}\left(D_{j}^{c}\right)\right]=Z_{m}\left(Q_{i}\right)-Z_{m}\left(Q_{j}\right) \pm \alpha m}
\end{aligned}
$$

where $\alpha=0,1$. The condition for the value $\pm \beta n$ follows similarly. Likewise for the lepton sector, one obtains

$$
V_{i j}^{l} \sim \lambda^{F_{e f f}\left(L_{i}\right)-F_{e f f}\left(L_{j}\right) \pm \alpha m \pm \beta n}
$$

Therefore, the masses and mixing angles can be enhanced or suppressed by $\lambda^{ \pm m \pm n}$ relative to the scaling predictions obtained when the family symmetry is the continuous flavor symmetry $U(1) \times U(1)^{\prime} \times U(1)^{\prime \prime}$ because of the discrete nature of $Z_{m} \times Z_{n}$. Note that in the case where the light neutrino masses are generated by the type I seesaw mechanism and all fermion charges are positive, the neutrino masses and mixing angles still do not depend on the details of the right-handed neutrino sector, except for the possible enhancement or suppression associated to the $Z_{m} \times Z_{n}$ flavor symmetry.

Furthermore, when the flavor symmetry is reduced to $U(1) \times Z_{m}$ by taking $n=0$, all the above results remain valid. It is remarkable that we can employ the $U(1) \times Z_{m}$ flavor symmetry to maintain the $\mathrm{BL}_{2}$ mixing while achieving very different neutrino masses without fine-tuning. We shall restrict our attention to the case of a $Z_{2}$ symmetry which is the minimal nontrivial $Z_{m}$ group (see, for example, the explicit model construction given in sec. 4 below). In this case just the $Z_{m}$ symmetry can reproduce a hierarchy in neutrino masses of order $\lambda^{2}$ consistent with the observed ratio of solar-to-atmospheric splittings.

In contrast, note that since the reactor neutrino mixing is necessarily of order $\sin \theta_{13} \sim$ $\lambda^{2 \pm \alpha m}$ the $U(1) \times Z_{m}$ flavor symmetry can not produce the $\mathrm{BL}_{1}$ mixing pattern. Indeed for such $\mathrm{BL}_{1}$ texture one has $\sin \theta_{12} \sim \lambda$ and $\sin \theta_{23} \sim \lambda$, which is in conflict with the required linear behavior of the reactor mixing angle $\sin \theta_{13} \sim \lambda$. Note parenthetically that the $Z_{1}$ group consists of only the identity element, so the group $U(1) \times Z_{1}$ is isomorphic to $U(1)$, and the $Z_{1}$ charge of field is 0 , hence the flavor symmetry $U(1) \times Z_{1}$ produces a wrong scaling behavior $\sin \theta_{13} \sim \lambda^{2}$.

We now turn to the realistic case of the $U(1) \times Z_{m} \times Z_{n}$ flavor symmetry. If both solar and atmospheric neutrino mixing angles are of order $\lambda$ then the reactor angle would be 
constrained to be of order $\sin \theta_{13} \sim \lambda^{2 \pm \alpha m \pm \beta n}$. As a result, one can have $\sin \theta_{13} \sim \lambda$ if the parity of $m$ and $n$ is opposite. This is an interesting observation of the present work. In section 3, a concrete model for the $\mathrm{BL}_{1}$ mixing pattern is presented based on the flavor symmetry $U(1) \times Z_{3} \times Z_{4}$.

Since an Abelian flavor symmetry can not predict the exact value of the $\mathcal{O}(1)$ coefficients in front of each invariant operator, we must content ourselves with explaining the orders of magnitude of fermion masses and flavor mixing parameters. To identify the phenomenologically acceptable mass matrices, we will estimate the various mass ratios and mixing angles as approximate powers of the small parameter $\lambda$. The hierarchies in the quark mixing angles are clearly displayed in Wolfenstein's truncated form [21] of the parametrization of the CKM matrix [22]:

$$
V_{C K M}=\left(\begin{array}{ccc}
1-\lambda^{2} / 2 & \lambda & A \lambda^{3}(\rho-i \eta) \\
-\lambda & 1-\lambda^{2} / 2 & A \lambda^{2} \\
A \lambda^{3}(1-\rho-i \eta) & -A \lambda^{2} & 1
\end{array}\right)
$$

where the quantities $A, \rho$ and $\eta$ are experimentally determined to be of order one. Therefore the order of magnitude of the three mixing angles is given in terms of the $\lambda$ as

$$
\left|V_{u s}\right| \sim \lambda, \quad\left|V_{c b}\right| \sim \lambda^{2}, \quad\left|V_{u b}\right| \sim \lambda^{3}-\lambda^{4}
$$

The charged fermion mass ratios at the grand unified theory (GUT) scale should satisfy [23]

$$
\begin{aligned}
\frac{m_{u}}{m_{c}} & \sim \lambda^{4}, & \frac{m_{c}}{m_{t}} & \sim \lambda^{3}-\lambda^{4}, \\
\frac{m_{d}}{m_{s}} & \sim \lambda^{2}, & \frac{m_{s}}{m_{b}} & \sim \lambda^{2}, \\
\frac{m_{e}}{m_{\mu}} & \sim \lambda^{2}-\lambda^{3}, & \frac{m_{\mu}}{m_{\tau}} & \sim \lambda^{2}
\end{aligned}
$$

as well as

$$
\frac{m_{b}}{m_{\tau}} \sim 1, \quad \frac{m_{b}}{m_{t}} \sim \lambda^{3}
$$

for the intrafamily hierarchy. The first identity is the well-known $b-\tau$ unification relation. For the neutrinos, we required that the lepton mixing is of $\mathrm{BL}_{1}$ or $\mathrm{BL}_{2}$ type depending on the octant of $\theta_{23}$. For the quark sector, all the explicit models are properly constructed to meet the requirement $m_{t} / v_{u} \sim 1$ and $m_{b} / v_{d} \sim \lambda^{3}$

\section{Model for $\mathrm{BL}_{1}$ mixing}

As has been shown in the previous section, one can reproduce the $\mathrm{BL}_{1}$ texture within the framework of $U(1) \times Z_{m} \times Z_{n}$ family symmetry, where $m$ and $n$ should have different parity. For concreteness, we shall use $m=3$ and $n=4$ for our model. For such symmetry choice the possible model realization of the $\mathrm{BL}_{1}$ texture is not unique. As a concrete example, here the horizontal charges of the lepton fields are taken to be

$$
\begin{aligned}
& L_{1}:(4,1,3), \quad L_{2}:(3,2,2), \quad L_{3}:(1,1,1) \text {, } \\
& E_{1}^{c}:(3,2,2), \quad E_{2}^{c}:(1,2,2), \quad E_{3}^{c}:(0,0,0) \text {. }
\end{aligned}
$$


One immediately obtains the charged lepton mass matrix

$$
M_{e} \sim\left(\begin{array}{ccc}
\lambda^{8} & \lambda^{6} & \lambda^{8} \\
\lambda^{7} & \lambda^{5} & \lambda^{7} \\
\lambda^{7} & \lambda^{5} & \lambda^{3}
\end{array}\right) v_{d}
$$

which yields the mass ratios

$$
\frac{m_{e}}{m_{\mu}} \sim \lambda^{3}, \quad \frac{m_{\mu}}{m_{\tau}} \sim \lambda^{2},
$$

that are consistent with the experimental requirements. For the charged assignments in Eq.(26), the light neutrino mass matrix is given by

$$
M_{\nu} \sim\left(\begin{array}{ccc}
\lambda^{12} & \lambda^{8} & \lambda^{7} \\
\lambda^{8} & \lambda^{7} & \lambda^{7} \\
\lambda^{7} & \lambda^{7} & \lambda^{6}
\end{array}\right) \frac{v_{u}^{2}}{\Lambda} .
$$

It predicts the light neutrino mass eigenvalues as follows:

$$
m_{1} \sim \lambda^{8} \frac{v_{u}^{2}}{\Lambda}, \quad m_{2} \sim \lambda^{7} \frac{v_{u}^{2}}{\Lambda}, \quad m_{3} \sim \lambda^{6} \frac{v_{u}^{2}}{\Lambda}
$$

The neutrino mass spectrum is normal hierarchy, this is confirmed by subsequent numerical analysis. It is remarkable that this model gives rise to $m_{2} / m_{3} \sim \lambda$ and $\Delta m_{\text {sol }}^{2} / \Delta m_{\text {atm }}^{2} \sim$ $\lambda^{2}$, which is in excellent agreement with the experimental data. In conventional $U(1)$ or $U(1) \times Z_{m}$ flavor symmetries, if any ratio between neutrino masses is an odd power of the small breaking parameter, generally the mixing angle between the two neutrinos will vanish 25]. The crucial point is that the element $\left(M_{\nu}\right)_{22}$, which would have been $\mathcal{O}\left(\lambda^{14}\right)$ under the continuous $U(1) \times U(1)^{\prime} \times U(1)^{\prime \prime}$ symmetry, is enhanced to $\mathcal{O}\left(\lambda^{7}\right)$ due to the discrete symmetry $Z_{3} \times Z_{4}$. Diagonalizing the mass matrices in Eq.(27) and Eq.(29) by the standard perturbative techniques described in Refs. [18,25, 26], we get the three lepton flavor mixing angles

$$
\sin \theta_{12} \sim \lambda, \quad \sin \theta_{13} \sim \lambda, \quad \sin \theta_{23} \sim \lambda
$$

Hence the $\mathrm{BL}_{1}$ pattern is produced automatically. Note that the solar neutrino mixing $\sin \theta_{12}$ arises from order $\lambda$ contributions from the diagonalization of both $M_{e}$ and $M_{\nu}$, while at leading order the reactor and the atmospheric neutrino mixing angles receive contribution only from the neutrino mass matrix $M_{\nu}$. The off-diagonal elements $\left(M_{\nu}\right)_{13}$ and $\left(M_{\nu}\right)_{23}$ are enhanced by $Z_{4}$ and $Z_{3}$ respectively, hence we have $\sin \theta_{13} \sim \lambda$ and $\sin \theta_{23} \sim \lambda$ instead of the naive expectations $\sin \theta_{13} \sim \lambda^{5}$ and $\sin \theta_{23} \sim \lambda^{4}$ characteristic of the continuous flavor symmetry case.

In the following, we shall extend the model to encompass also quark sector. Since GUT relates quarks and leptons, the transformation properties of quark fields can be determined from those of leptons. In order to give a successful description of the observed fermion mass hierarchies and mixings simultaneously under the same flavor symmetry acting on quarks and leptons we work in the framework of $S U(5)$, for definiteness. Another motivation of 
considering $S U(5)$ unification is the anomaly cancellation. If the $U(1)$ flavor symmetry is gauged then a general assignment of flavor charges to the fields will be anomalous. One can imagine the anomaly to be canceled via the Green-Schwarz mechanism [27], however, one must check whether the correct relations are satisfied [28]. A convenient way to ensure that the flavor charges are amenable to cancellation is to have the flavor symmetry to commute with the $S U(5)$ group [29].

Here we propose a model with the quark and lepton matter assignments manifestly compatible with potential unification within $S U(5)$. A complete study of a realistic grand unified model model addressing the well-known problems such as the doublet-triplet splitting, the proton lifetime and gauge coupling unification, is beyond the scope of the present paper and will be studied elsewhere.

In the conventional $S U(5)$ grand unified theory, the fields $D_{i}^{c}$ and $L_{i}$ of the same generation are assigned to a $\overline{\mathbf{5}}$ multiplet, the fields $Q_{i}, U_{i}^{c}$ and $E_{i}^{c}$ are unified in the 10 representation. Since the flavor symmetry is required to commute with the gauge symmetry, this means that the fields in each gauge multiplet transform in the same way under the flavor symmetry. Consequently, the quantum numbers of the quark fields under the flavor symmetry $U(1) \times Z_{3} \times Z_{4}$ are as follows:

$$
\begin{array}{lll}
Q_{L 1}:(3,2,2), & Q_{L 2}:(1,2,2), & Q_{L 3}:(0,0,0), \\
U_{1}^{c}:(3,2,2), & U_{2}^{c}:(1,2,2), & U_{3}^{c}:(0,0,0), \\
D_{1}^{c}:(4,1,3), & D_{2}^{c}:(3,2,2), & D_{3}^{c}:(1,1,1) .
\end{array}
$$

We note that although there are many possible assignments to produce the $\mathrm{BL}_{1}$ texture in the neutrino sector, only a few of them can satisfy the quark sector phenomenological constraints within $S U(5)$. It is well-known that the minimal $S U(5)$ grand unified theory predicts that the down-type quark mass matrix is the transpose of the charged lepton mass matrix, therefore the down-type quarks and charged lepton masses are closely related : $m_{e}=m_{d}, m_{\mu}=m_{s}$ and $m_{\tau}=m_{b}$, which are in gross disagreement with the measured fermion masses and must be corrected [30]. This can be done through the contribution of renormalizable [30] or nonrenormalizable [31] operators to the Yukawa matrices. Following Ref. [32], we introduce an additional $U(1) \times Z_{3} \times Z_{4}$ singlet superfield $\Sigma$ transforming as a 75 of $S U(5)$, which has non-renormalizable couplings to fermions of the form $\overline{5} \mathbf{1 0} H_{\overline{5}} \Sigma / \Lambda$. The Yukawa couplings of the down-type quark and charged leptons then arise from the two $S U(5) \times U(1) \times Z_{3} \times Z_{4}$ invariant superpotential terms 2 .

$$
\begin{aligned}
W_{d} & =\left(\mathbf{1 0}_{i}\left(C_{1}\right)_{i j} \overline{\mathbf{5}}_{j} H_{\overline{\mathbf{5}}}+\frac{\Sigma}{\Lambda} \mathbf{1 0}_{i}\left(C_{2}\right)_{i j} \overline{\mathbf{5}}_{j} H_{\overline{\mathbf{5}}}\right)\left(\frac{\Theta_{1}}{\Lambda}\right)^{F\left(\mathbf{1 0}_{i}\right)+F\left(\overline{\mathbf{5}}_{j}\right)}\left(\frac{\Theta_{2}}{\Lambda}\right)^{\left[Z_{3}\left(\mathbf{1 0}_{i}\right)+Z_{3}\left(\overline{\mathbf{5}}_{j}\right)\right]} \\
& \times\left(\frac{\Theta_{3}}{\Lambda}\right)^{\left[Z_{4}\left(\mathbf{1 0}_{i}\right)+Z_{4}\left(\overline{\mathbf{5}}_{j}\right)\right]}
\end{aligned}
$$

${ }^{2}$ The $\mathbf{7 5}$ could in principle also give a contribution in the up sector. However, following Ref. [32] we neglect such a term since it is not needed to reproduce the up-type quark masses. 
which, after the scalar components of $\Sigma$ acquires a VEV, lead to:

$$
\begin{aligned}
& \left(\mathbf{Y}_{d}\right)_{i j}=\left(\left(C_{1}\right)_{i j}+\kappa\left(C_{2}\right)_{i j}\right) \lambda^{F\left(Q_{i}\right)+F\left(D_{j}^{c}\right)+\left[Z_{3}\left(Q_{i}\right)+Z_{3}\left(D_{j}^{c}\right)\right]+\left[Z_{4}\left(Q_{i}\right)+Z_{4}\left(D_{j}^{c}\right)\right]} \\
& \left(\mathbf{Y}_{e}\right)_{i j}=\left(\left(C_{1}\right)_{i j}-3 \kappa\left(C_{2}\right)_{i j}\right) \lambda^{F\left(Q_{j}\right)+F\left(D_{i}^{c}\right)\left[Z_{3}\left(Q_{j}\right)+Z_{3}\left(D_{i}^{c}\right)\right]+\left[Z_{4}\left(Q_{j}\right)+Z_{4}\left(D_{i}^{c}\right)\right]},
\end{aligned}
$$

where $\kappa=\langle\Sigma\rangle / \Lambda$, which breaks the transposition relation between $\mathbf{Y}_{d}$ and $\mathbf{Y}_{e}$ and can explain the difference between down-type quarks and charged lepton masses. In our numerical fits, we take $\kappa=0.3$ for illustration and find that realistic values for down-type quarks and charged lepton masses can be reproduced. The superpotential for the up-type quark mass is

$$
W_{u}=\mathbf{1 0}_{i}\left(C_{3}\right)_{i j} \mathbf{1 0}_{j} H_{5}\left(\frac{\Theta_{1}}{\Lambda}\right)^{F\left(\mathbf{1 0}_{i}\right)+F\left(\mathbf{1 0}_{j}\right)}\left(\frac{\Theta_{2}}{\Lambda}\right)^{\left[Z_{3}\left(\mathbf{1 0}_{i}\right)+Z_{4}\left(\mathbf{1 0}_{j}\right)\right]}\left(\frac{\Theta_{3}}{\Lambda}\right)^{\left[Z_{4}\left(\mathbf{1 0}_{i}\right)+Z_{4}\left(\mathbf{1 0}_{j}\right)\right]},
$$

where one has $\left(C_{3}\right)_{i j}=\left(C_{3}\right)_{j i}$ due to the constraint of the $S U(5)$ gauge symmetry. Then one can express the effective Yukawa couplings for the up-type quark in terms of the flavor symmetry charges as

$$
\left(\mathbf{Y}_{u}\right)_{i j}=\left(C_{3}\right)_{i j} \lambda^{F\left(Q_{i}\right)+F\left(Q_{j}\right)+\left[Z_{3}\left(Q_{i}\right)+Z_{3}\left(Q_{j}\right)\right]+\left[Z_{4}\left(Q_{i}\right)+Z_{4}\left(Q_{j}\right)\right]}
$$

With the assignments dictated by Eq.(32), one has the following patterns for the up- and down-type quark mass matrices,

$$
M_{u} \sim\left(\begin{array}{ccc}
\lambda^{7} & \lambda^{5} & \lambda^{7} \\
\lambda^{5} & \lambda^{3} & \lambda^{5} \\
\lambda^{7} & \lambda^{5} & 1
\end{array}\right) v_{u}, \quad M_{d} \sim\left(\begin{array}{ccc}
\lambda^{8} & \lambda^{7} & \lambda^{7} \\
\lambda^{6} & \lambda^{5} & \lambda^{5} \\
\lambda^{8} & \lambda^{7} & \lambda^{3}
\end{array}\right) v_{d}
$$

which yield

$$
\left|V_{u s}\right| \sim \lambda^{2}, \quad\left|V_{c b}\right| \sim \lambda^{2}, \quad\left|V_{u b}\right| \sim \lambda^{4} .
$$

We note that both the up and down quark sector contribute $\lambda^{2}$ to the mixing element $\left|V_{u s}\right|$, therefore an accidental enhancement of $\mathcal{O}\left(\lambda^{-1}\right)$ among the undetermined order one coefficients $\left(C_{1}\right)_{i j},\left(C_{2}\right)_{i j}$ and $\left(C_{3}\right)_{i j}$ is required in order to describe the correct Cabibbo angle. The remaining CKM mixing angles $\left|V_{c b}\right|$ and $\left|V_{u b}\right|$ arise solely from the diagonalization of the down-type quark mass matrix $M_{d}$. In addition, the pattern given by Eq.(37) leads to the following quark mass scalings:

$$
\begin{aligned}
& m_{u} \sim \lambda^{7} v_{u}, \quad m_{c} \sim \lambda^{3} v_{u}, \quad m_{t} \sim v_{u}, \\
& m_{d} \sim \lambda^{8} v_{d}, \quad m_{s} \sim \lambda^{5} v_{d}, \quad m_{b} \sim \lambda^{3} v_{d},
\end{aligned}
$$

which describe the experimental data satisfactorily. Note that the second term in Eq.(33) accounts for the mass difference between the down-type quarks and charged leptons, allowing for an acceptable charged fermion mass pattern.

In order to see in a quantitative way how well the model describes the observed values of the fermion masses and mixings, we perform a numerical analysis, within three independent 
different seeding methods: namely flat, Gaussian and exponential distributions. The modulus of the undetermined order one coefficients are taken to be random numbers with flat, Gaussian and exponential distributions in turn, the corresponding phases are varied between 0 and $2 \pi$. The probability density function $f(x)$ of the three distributions is well known

$$
f(x)=\left\{\begin{array}{cc}
\frac{1}{b-a} & a \leq x \leq b \\
0 & x<a \text { or } x>b
\end{array} .\right.
$$

For flat distribution, we take $a=1 / 3$ and $b=3$ for illustration in the present work. In the case of gaussian distribution,

$$
f(x)=\frac{a}{\sqrt{2 \pi} \sigma} e^{-\frac{(x-\mu)^{2}}{2 \sigma^{2}}} .
$$

We set the mean $\mu=1$ and the standard deviation $\sigma=1.5$ in our numerical calculation. The probability density function for the exponential distribution is

$$
f(x)=\left\{\begin{array}{cc}
\lambda e^{-\lambda x} & x \geq 0 \\
0 & x<0
\end{array} .\right.
$$

Its statistic mean is $1 / \lambda$, and $\lambda$ is taken to be 1 as a typical value for numerical simulation. To the extent that our results are independent of the choice of seeding method, they are robust and not simply an artifact of the choice of the seed function.

The coefficients $\left(C_{1}\right)_{i j},\left(C_{2}\right)_{i j},\left(C_{3}\right)_{i j}$ and $\left(y_{\nu}\right)_{i j}$ are treated as random complex numbers with arbitrary phases and absolute value in the interval of $[1 / 3,3]$. Then we calculate the quark and lepton masses as well as the CKM and lepton mixing matrix entries which are required to lie in the experimentally allowed ranges. The numerical results are found to be nicely consistent with the above theoretical estimates and qualitative discussions. Since the flavor parameters of the quark sector are precisely measured, here we focus on the neutrino sector. As an example the predicted distributions for the light neutrino masses and atmospheric mixing parameter are shown in Fig. 1. The light neutrino masses follow the normal hierarchy pattern and, for all the points produced, though all non-vanishing, they are rather tiny, with most of the expected $m_{1}$ values below $0.015 \mathrm{eV}$. As to the mixing angles, no specific values of $\theta_{12}$ and $\theta_{13}$ are favored within $3 \sigma$, and hence they are not shown in the figure 3 . In contrast, however, the atmospheric neutrino mixing angle $\theta_{23}$ obeys $\sin ^{2} \theta_{23}<1 / 2$, which means that non-maximal $\theta_{23}$ values are preferred, as indicated by current neutrino oscillation global analyses post-Neutrino 2012 [6] 8], with a preference for the first octant. This has been one of our motivations for introducing $\mathrm{BL}_{1}$ mixing pattern, which leads to $\sin \theta_{23}$ of order $\lambda$ at leading order.

The rare process, neutrinoless double beta decay $(0 \nu 2 \beta)$, constitutes an important probe for the Majorana nature of neutrino and lepton number violation [33], a sizable number of new experiments are currently running, under construction, or in the planing phase. The

\footnotetext{
${ }^{3}$ Similarly, we can hardly see any specific preferred pattern for the CP violating phases $\delta, \varphi_{1}$ and $\varphi_{2}$, hence, as before, these are not shown.
} 

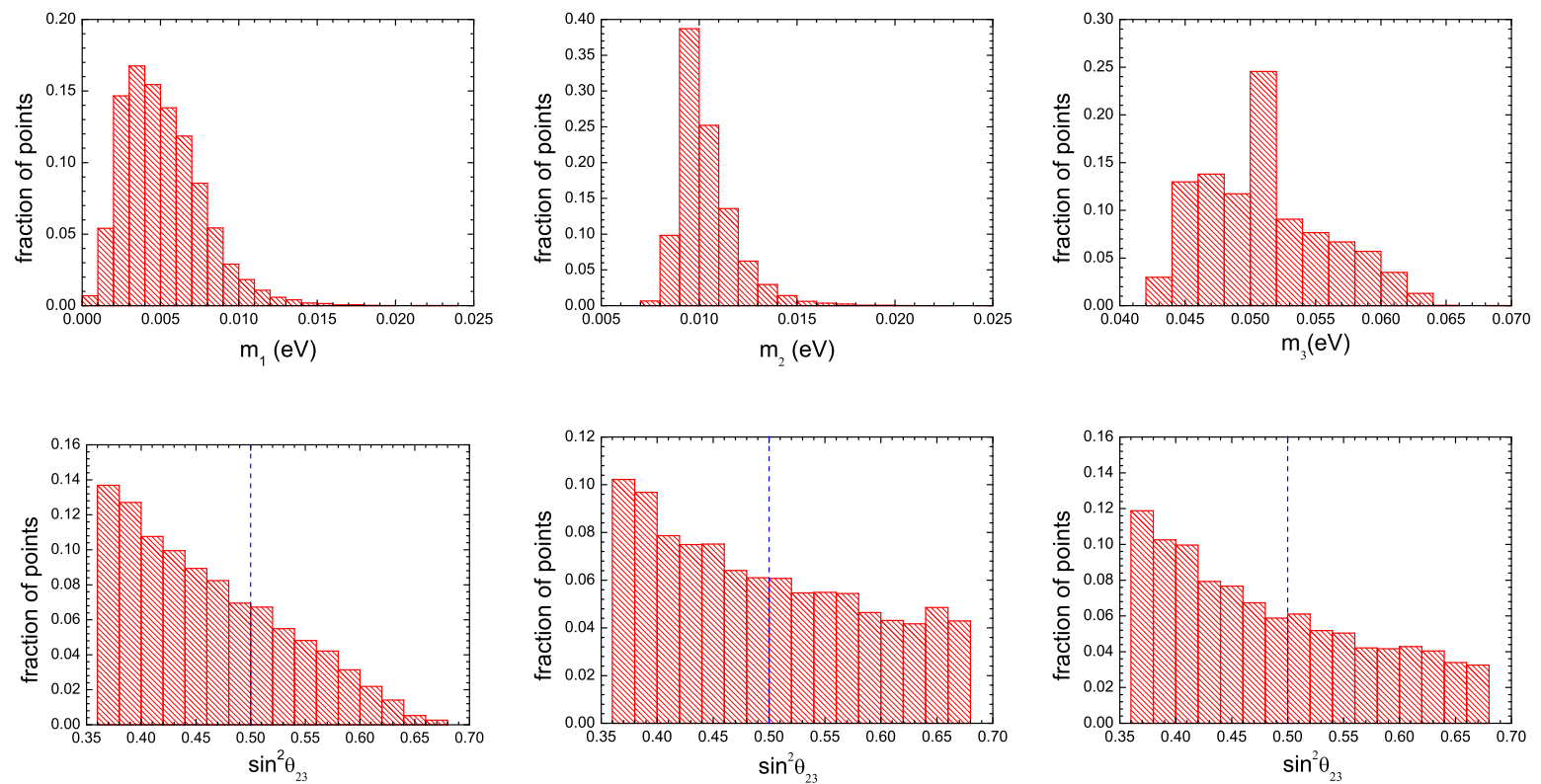

Figure 1: Histograms for the distribution of light neutrino masses and atmospheric neutrino mixing parameter in the $\mathrm{BL}_{1}$ model. In the second row, the left, middle and right panels are obtained with using different seed procedures for the order one Yukawa coefficients, namely flat, exponential and Gaussian, respectively, from left to right.

histogram for the distribution of the effective $0 \nu 2 \beta$-decay mass $\left|m_{e e}\right|$ and its correlation with the lightest neutrino mass $m_{1}$ are given in Fig. 2. We also show the future sensitivity on the lightest neutrino mass of $0.2 \mathrm{eV}$ from the KATRIN experiment [34]. The horizontal lines represent the sensitivities of the future $0 \nu 2 \beta$-decay experiments CUORE [35] and MAJORANA [36]/GERDA III [37], which are approximately $18 \mathrm{meV}$ and $12 \mathrm{meV}$ respectively. Clealry the expected effective mass $\left|m_{e e}\right|$ is predicted to be far below the sensitivities of the planned $0 \nu 2 \beta$ experiments. The reason for this is the strong destructive interference amongst the three light neutrinos, as seen in the right panel. As a result, if $0 \nu 2 \beta$ decay will be detected in the near future, our construction would be ruled out.

To keep our discussion as generic as possible, we describe the light neutrino masses by the effective higher-dimensional Weinberg operators as shown in Eq.(3) and Eq.(12), which could come from the so-called type I seesaw mechanism, by integrating out the right-handed neutrinos. It is interesting to note that $U(1)$ flavor symmetry models have particularly simple factorization properties [24,25]: our various predictions for the light neutrino parameters given above, are independent of the $U(1)$ charge assignments of the right-handed neutrinos. For example, suppose we introduce three right-handed neutrinos transforming under the flavor symmetry $U(1) \times Z_{3} \times Z_{4}$ as follows:

$$
N_{1}^{c}:\left(n_{1}, 0,1\right), \quad N_{2}^{c}:\left(n_{2}, 0,3\right), \quad N_{3}^{c}:\left(n_{3}, 2,2\right)
$$

where $n_{i}$, which are positive integers denoting the $U(1)$ charges of the heavy Majorana neutrinos. Then one can straightforwardly read out the Dirac neutrino mass matrix $M_{D}$ and 
the Majorana mass matrix $M_{N}$ of the right-handed neutrinos,

$$
M_{D} \sim\left(\begin{array}{ccc}
\lambda^{5+n_{1}} & \lambda^{7+n_{2}} & \lambda^{5+n_{3}} \\
\lambda^{8+n_{1}} & \lambda^{6+n_{2}} & \lambda^{4+n_{3}} \\
\lambda^{4+n_{1}} & \lambda^{2+n_{2}} & \lambda^{4+n_{3}}
\end{array}\right) v_{u}, \quad M_{N} \sim\left(\begin{array}{ccc}
\lambda^{2+2 n_{1}} & \lambda^{n_{1}+n_{2}} & \lambda^{5+n_{1}+n_{3}} \\
\lambda^{n_{1}+n_{2}} & \lambda^{2+2 n_{2}} & \lambda^{3+n_{2}+n_{3}} \\
\lambda^{5+n_{1}+n_{3}} & \lambda^{3+n_{2}+n_{3}} & \lambda^{1+2 n_{3}}
\end{array}\right) \Lambda .
$$

The resulting effective light neutrino mass matrix is given by the seesaw formula

$$
M_{\nu}=-M_{D} M_{N}^{-1} M_{D}^{T} \sim\left(\begin{array}{ccc}
\lambda^{9} & \lambda^{8} & \lambda^{7} \\
\lambda^{8} & \lambda^{7} & \lambda^{7} \\
\lambda^{7} & \lambda^{7} & \lambda^{6}
\end{array}\right) \frac{v_{u}^{2}}{\Lambda}
$$

This is the same as obtained in the above effective approach given in Eq.(29) except that the smallest element $\left(M_{\nu}\right)_{11}$ is of order $\lambda^{9}$ instead of $\lambda^{12}$, both of them are too small to affect the predictions for the neutrino oscillation parameters. We get the same light neutrino masses in Eq.(30) and the same neutrino mixing angles in Eq.(31) as in the above effective Weinberg operator neutrino mass generation. We would like to emphasize again that the predictions for the neutrino masses and mixing parameters are independent of the charges $n_{i}$, which drop out in the seesaw formula for the light neutrino mass matrix. However, different values of the charges $n_{i}$ obviously give rise to different Dirac neutrino Yukawa coupling $Y_{\nu} \equiv M_{D} / v_{u}$. As a result, the predictions for charged lepton flavor violation (LFV) processes such as $\mu \rightarrow e \gamma$, $\tau \rightarrow \mu \gamma$ and $\mu \rightarrow 3 e$ are quite different [38]. Recalling that the branching ratio of the LFV process is generally proportional to $Y_{\nu}^{4}$, the stringent bound on LFV, in particular from $\mu \rightarrow e \gamma$, can be easily satisfied for only slightly large $n_{i}$ [38] while keeping the predictions for neutrino parameters intact.

\section{Model for $\mathrm{BL}_{2}$ mixing}

As explained in section 2, the order one atmospheric neutrino mixing $\sin \theta_{23} \sim 1$ generically implies that the corresponding masses of $\nu_{2}$ and $\nu_{3}$ are of the same order of magnitude within pure $U(1)$ family symmetry schemes. As a result, the neutrino mass spectrum is quasi-degenerate and strong fine-tuning is required in order to account for the measured mass-squared differences $\Delta m_{\text {sol }}^{2}$ and $\Delta m_{\text {atm }}^{2}$. Furthermore, the renormalization group evolution effects could drastically enhance the neutrino mixing angles due to the degeneracy, so that the $\mathrm{BL}_{2}$ texture would be spoiled at the electroweak scale. This can be avoided by extending the flavor symmetry to $U(1) \times Z_{m}$. Now the whole flavor symmetry is chosen to be $U(1) \times Z_{2}$, the lepton fields carry the following $U(1) \times Z_{2}$ charges:

$$
\begin{aligned}
& L_{1}:(3,0), \quad L_{2}:(3,1), \quad L_{3}:(2,0) \text {, } \\
& E_{1}^{c}:(4,0), \quad E_{2}^{c}:(2,1), \quad E_{3}^{c}:(0,1) \text {, }
\end{aligned}
$$



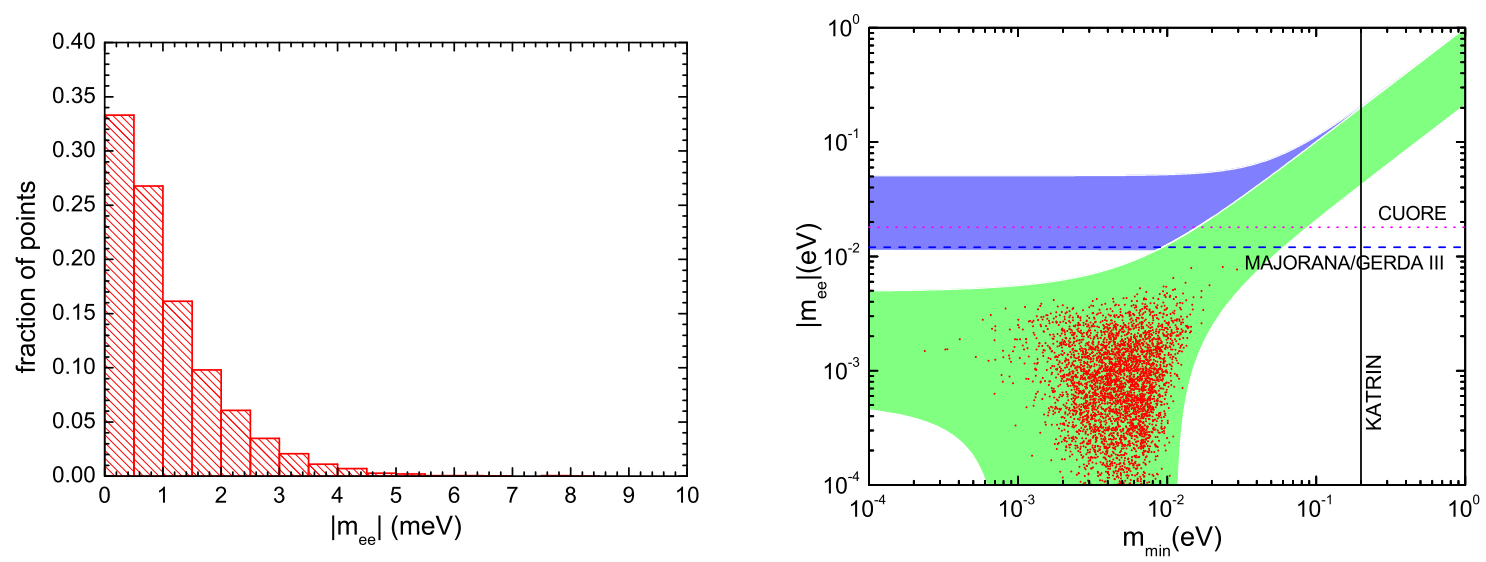

Figure 2: Histogram of the effective mass $\left|m_{e e}\right|$ (left panel) and the scatter plot of $\left|m_{e e}\right|$ versus the lightest neutrino mass $m_{1}$ (right panel) for the $\mathrm{BL}_{1}$ model. The colored bands represent the regions for the $3 \sigma$ ranges of the oscillation parameters in the normal and inverted neutrino mass spectrum respectively. The future sensitivity of $0.2 \mathrm{eV}$ of the KATRIN experiment is shown by the vertical solid line, while the future expected bounds on $\left|m_{e e}\right|$ from the CUORE and MAJORANA/GERDA III experiments are represented by horizontal lines.

Then the light neutrino mass matrix is given, apart from the order one coefficients, as

$$
M_{\nu} \sim\left(\begin{array}{ccc}
\lambda^{6} & \lambda^{7} & \lambda^{5} \\
\lambda^{7} & \lambda^{6} & \lambda^{6} \\
\lambda^{5} & \lambda^{6} & \lambda^{4}
\end{array}\right) \frac{v_{u}^{2}}{\Lambda},
$$

which yields

$$
m_{1} \sim \lambda^{6} v_{u}^{2} / \Lambda, \quad m_{2} \sim \lambda^{6} v_{u}^{2} / \Lambda, \quad m_{3} \sim \lambda^{4} v_{u}^{2} / \Lambda
$$

One sees that the first two light neutrinos are quasi-degenerate in this model, and their masses are suppressed by $\mathcal{O}\left(\lambda^{2}\right)$ with respect to the third one. This prediction is consistent with the observation that the solar neutrino mass difference $\Delta m_{\mathrm{sol}}^{2}$ is much smaller than the atmospheric neutrino mass difference $\Delta m_{\mathrm{atm}}^{2}$. Moreover, the neutrino mass spectrum is predicted to be of the normal hierarchy type here, the same as in the previous $\mathrm{BL}_{1}$ model (this is also confirmed our numerical analysis). The next generation of higher precision neutrino oscillation experiments is designed to be able to measure neutrino mass hierarchy and the CP phase [39]. Should the latter be determined to be of the inverted type by future experiments, both of our models would be ruled out. On the other hand, the charged lepton mass matrix takes the following form:

$$
M_{e} \sim\left(\begin{array}{ccc}
\lambda^{7} & \lambda^{6} & \lambda^{4} \\
\lambda^{8} & \lambda^{5} & \lambda^{3} \\
\lambda^{6} & \lambda^{5} & \lambda^{3}
\end{array}\right) v_{d}
$$

which has a "lopsided" structure, a large 2-3 mixing arises from the diagonalization of $M_{e}$. Obviously it also gives the correct order of magnitude for the charged lepton mass ratios. 
Combining the contribution from both the neutrino and the charged lepton mass matrices diagonalization, the leptonic mixing angles are given by

$$
\sin \theta_{12} \sim \lambda, \quad \sin \theta_{13} \sim \lambda \quad \sin \theta_{23} \sim 1
$$

This is exactly the desired $\mathrm{BL}_{2}$ mixing pattern, Eq. (2). Here we would like to point out that since the Super-Kamiokande data indicted large atmospheric neutrino mixing, perhaps even maximal [41], there have been several attempts to account for the large atmospheric neutrino mixing $\sin \theta_{23} \sim 1$ in terms of Abelian flavor symmetries [40]. However, it was usually assumed that the reactor angle $\theta_{13}$ was rather small, at most of order $\lambda^{2}$ at that time [42]. In contrast, in our construction the consistency between large $\sin \theta_{23}$ and sizeable $\sin \theta_{13}$ mixing angles emerges naturally.

In what follows, we extend the model to include quarks within the $S U(5)$ unified framework. The fields $Q_{i}$ and $U_{i}^{c}$ together with $E_{i}^{c}$ within the same generation fill out the 10 representation, while $D_{i}^{c}$ and the left-handed lepton doublet $L_{i}$ make up the $\overline{\mathbf{5}}$ representation. As a result, we can determine the transformation properties of the quark fields under the $U(1) \times Z_{2}$ flavor symmetry as follows:

$$
\begin{array}{lll}
Q_{1}:(4,0), & Q_{2}:(2,1), & Q_{3}:(0,1), \\
U_{1}^{c}:(4,0), & U_{2}^{c}:(2,1), & U_{3}^{c}:(0,1), \\
D_{1}^{c}:(3,0), & D_{2}^{c}:(3,1), & D_{3}^{c}:(2,0) .
\end{array}
$$

The up and down quark mass matrices can be determined in a straightforward way as follows:

$$
M_{u} \sim\left(\begin{array}{ccc}
\lambda^{8} & \lambda^{7} & \lambda^{5} \\
\lambda^{7} & \lambda^{4} & \lambda^{2} \\
\lambda^{5} & \lambda^{2} & 1
\end{array}\right) v_{u}, \quad M_{d} \sim\left(\begin{array}{ccc}
\lambda^{7} & \lambda^{8} & \lambda^{6} \\
\lambda^{6} & \lambda^{5} & \lambda^{5} \\
\lambda^{4} & \lambda^{3} & \lambda^{3}
\end{array}\right) v_{d}
$$

which lead to

$$
\begin{aligned}
& \left|V_{u s}\right| \sim \lambda, \quad\left|V_{c b}\right| \sim \lambda^{2}, \quad\left|V_{u b}\right| \sim \lambda^{3}, \\
& \frac{m_{u}}{m_{c}} \sim \lambda^{4}, \quad \frac{m_{c}}{m_{t}} \sim \lambda^{4}, \quad \frac{m_{d}}{m_{s}} \sim \lambda^{2}, \quad \frac{m_{s}}{m_{b}} \sim \lambda^{2}, \quad \frac{m_{b}}{m_{t}} \sim \lambda^{3},
\end{aligned}
$$

which are in excellent agreement with observed quark mass hierarchies and CKM mixing angles. As in section 3, we perform a numerical simulation of the expected neutrino oscillation parameters. In Fig. 3 we display the resulting histograms for the neutrino mass eigenvalues. As expected on the basis of the qualitative estimate in Eq. (48), the light neutrino mass spectrum is normal hierarchy, the degenerate spectrum being strongly disfavored, and almost all the generated points lie in the region of the lightest neutrino mass $m_{1}$ smaller than $0.015 \mathrm{eV}$. The neutrinoless double beta decay predictions are shown in Fig. 4. One sees that, in contrast with the $\mathrm{BL}_{1}$ case, although the effective mass $\left|m_{e e}\right|$ is also quite small, with

\footnotetext{
${ }^{4}$ Insofar as the neutrino mixing angles $\theta_{i j}$ and CP phases $\delta, \varphi_{1}$ and $\varphi_{2}$ are concerned, we do not obtain any special predicted pattern, hence the results are not displayed.
} 

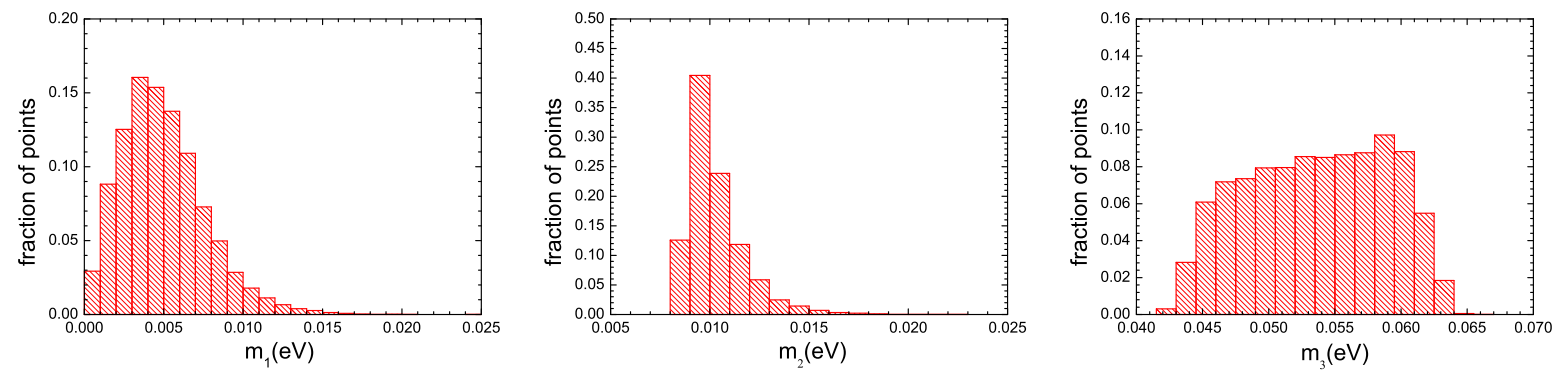

Figure 3: Light neutrino masses in the $\mathrm{BL}_{2}$ model.

$\left|m_{e e}\right|$ around $5 \mathrm{meV}$ preferred, there is a small portion of the parameter space of the model where the predictions for $\left|m_{e e}\right|$ approach the future experimental sensitivities. However, the points above the sensitivity limits on next generation experiments are statistically rather low. Therefore, if the signal of $0 \nu 2 \beta$ decay would be observed by upcoming experiments, the present $\mathrm{BL}_{2}$ model would also be ruled out, although not completely. We expect that the future $0 \nu 2 \beta$-decay experiments with sensitivity much higher than MAJORANA/GERDA III should be able to provide a better test of the model.

Now we turn to the seesaw realization of this model, the assignments for the right-handed neutrinos are not unique. As an example, we can introduce three right-handed neutrinos transforming as

$$
N_{1}^{c}:\left(n_{1}, 0\right), \quad N_{2}^{c}:\left(n_{2}, 1\right), \quad N_{3}^{c}:\left(n_{3}, 0\right) .
$$

Then we obtain the Dirac neutrino mass matrix $M_{D}$ as well as the right-handed neutrino mass matrix $M_{N}$,

$$
M_{D} \sim\left(\begin{array}{ccc}
\lambda^{3+n_{1}} & \lambda^{4+n_{2}} & \lambda^{3+n_{3}} \\
\lambda^{4+n_{1}} & \lambda^{3+n_{2}} & \lambda^{4+n_{3}} \\
\lambda^{2+n_{1}} & \lambda^{3+n_{2}} & \lambda^{2+n_{3}}
\end{array}\right) v_{u}, \quad M_{N} \sim\left(\begin{array}{ccc}
\lambda^{2 n_{1}} & \lambda^{1+n_{1}+n_{2}} & \lambda^{n_{1}+n_{3}} \\
\lambda^{1+n_{1}+n_{2}} & \lambda^{2 n_{2}} & \lambda^{1+n_{2}+n_{3}} \\
\lambda^{n_{1}+n_{3}} & \lambda^{1+n_{2}+n_{3}} & \lambda^{2 n_{3}}
\end{array}\right) \Lambda .
$$

The effective light neutrino mass matrix is given by the seesaw relation

$$
M_{\nu}=-M_{D} M_{M}^{-1} M_{D}^{T} \sim\left(\begin{array}{ccc}
\lambda^{6} & \lambda^{7} & \lambda^{5} \\
\lambda^{7} & \lambda^{6} & \lambda^{6} \\
\lambda^{5} & \lambda^{6} & \lambda^{4}
\end{array}\right) \frac{v_{u}^{2}}{\Lambda}
$$

This is exactly Eq.(47), consequently the predictions for neutrino parameters in Eq.(48) and Eq.(50) remain, note that dependence on the right-handed neutrino charges $n_{i}$ drops out. However, different values of the charges $n_{i}$ result in different LFV predictions, and the model would be less constrained for slightly large $n_{i}$ assignments [38].

\section{Conclusions}

The recent neutrino oscillation experimental highlights: (i) rather large value of reactor mixing angle $\theta_{13}$ and (ii) indication of significant deviation of the atmospheric neutrino 

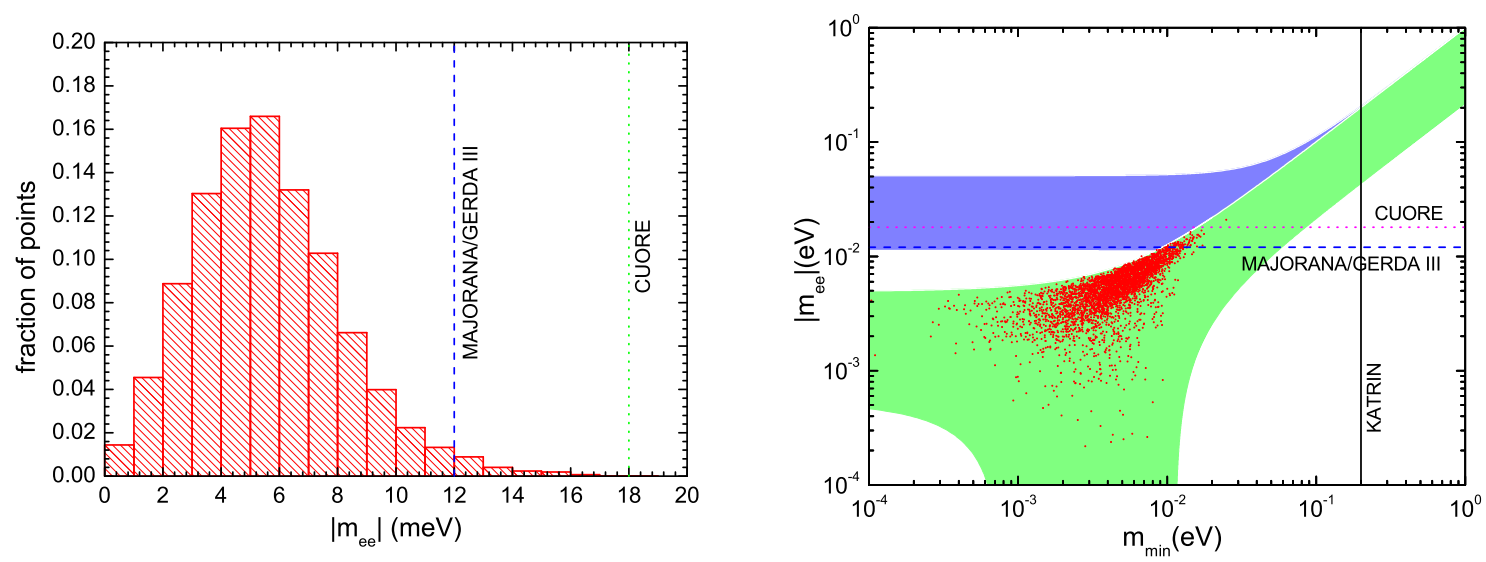

Figure 4: Histogram of the effective mass $\left|m_{e e}\right|$ (left panel) and the $\left|m_{e e}\right|$ versus the lightest neutrino mass $m_{1}$ correlation (right panel) predicted in the $\mathrm{BL}_{2}$ model.

mixing angle $\theta_{23}$ from maximality may change our theoretical approach for constructing neutrino mass models. In this paper, we study the Wolfenstein-like mixing schemes: $\mathrm{BL}_{1}$ mixing in which $\sin \theta_{12} \sim \lambda, \sin \theta_{13} \sim \lambda, \sin \theta_{23} \sim \lambda$, and $\mathrm{BL}_{2}$ mixing, in which $\sin \theta_{12} \sim \lambda$, $\sin \theta_{13} \sim \lambda, \sin \theta_{23} \sim 1$. The largish $\theta_{13}$ can be naturally accommodated in both of them, the two mixing patterns differ in the order of magnitude of $\sin \theta_{23}$, the $\mathrm{BL}_{1}$ texture is favored for $\theta_{23}$ in the first octant, while $\mathrm{BL}_{2}$ is preferred for the second octant $\theta_{23}$. In order to produce the $\mathrm{BL}_{1}$ mixing without invoking unnatural cancellation, the Abelian flavor symmetry should be $U(1) \times Z_{m} \times Z_{n}$ with the parity of $m$ and $n$ being opposite. A concrete model based on $U(1) \times Z_{3} \times Z_{4}$ family symmetry is constructed, where the light neutrino mass hierarchy $m_{2} / m_{3} \sim \lambda$ is realized due to the discrete nature of $Z_{3} \times Z_{4}$. The ratio $\Delta m_{\mathrm{sol}}^{2} / \Delta m_{\mathrm{atm}}^{2}$ is expected to be of order $\lambda^{2}$ in this model, which is in good agreement with experimental data in contrast with conventional $U(1)$ or $U(1) \times Z_{m}$ flavor symmetry constructions. Furthermore, the model is embedded into the $S U(5)$ grand unified theory to describe the quark masses and mixing simultaneously. As for the $\mathrm{BL}_{2}$ mixing, it can be reproduced within the framework of pure $U(1)$ flavor symmetry. However, the light neutrino mass spectrum is expected to be quasi-degenerate, hence fine-tuning of the neutrino mass parameters is needed in order to achieve the observed mass-squared differences. To improve upon this situation, the family symmetry is enlarged to $U(1) \times Z_{2}$, which gives rise to both large atmospheric neutrino mixing $\sin \theta_{23} \sim 1$ and hierarchical neutrino masses. The model is extended to $S U(5)$ grand unified theory as well.

We show that both models can give a successful description of the observed quark and lepton masses and mixing angles, and the numerical results are nicely in agreement with the theoretical estimates and the qualitative discussions. The light neutrinos are normal mass hierarchy in both models, quasi-degenerate spectrum is strongly disfavored. If the next generation high precision neutrino oscillation experiments determine that the neutrino mass spectrum is inverted hierarchy, both our constructions will be ruled out. The present 
framework can not predict the $\mathrm{CP}$ violating phases $\delta, \varphi_{1}$ and $\varphi_{2}$. The $0 \nu 2 \beta$-decay effective mass $\left|m_{e e}\right|$ is predicted to be rather small in both constructions, substantial part of the data are below the sensitivity of future experiments except for a region of the $\mathrm{BL}_{2}$ model indicated in Fig. 4. Therefore future $0 \nu 2 \beta$-decay experiments such as CUORE, MAJORANA and GERDA III will provide another important test of the present models.

\section{Acknowledgements}

This work was supported by the National Natural Science Foundation of China under Grant No 10905053, Chinese Academy KJCX2-YW-N29, DFG grant WI 2639/4-1 and the 973 project with Grant No. 2009CB825200; by the Spanish MINECO under grants FPA2011-22975 and MULTIDARK CSD2009-00064 (Consolider-Ingenio 2010 Programme), by Prometeo/2009/091 (Generalitat Valenciana), and by the EU ITN UNILHC PITN-GA2009-237920. S.M. is supported by a Juan de la Cierva grant.

\section{References}

[1] F. P. An et al. [DAYA-BAY Collaboration], Phys. Rev. Lett. 108, 171803 (2012) arXiv:1203.1669 [hep-ex]].

[2] J. K. Ahn et al. [RENO Collaboration], Phys. Rev. Lett. 108, 191802 (2012) arXiv:1204.0626 [hep-ex]].

[3] Y. Abe et al. [DOUBLE-CHOOZ Collaboration], Phys. Rev. Lett. 108, 131801 (2012) arXiv:1112.6353 [hep-ex]].

[4] K. Abe et al. [T2K Collaboration], Phys. Rev. Lett. 107, 041801 (2011) arXiv:1106.2822 [hep-ex]].

[5] P. Adamson et al. [MINOS Collaboration], Phys. Rev. Lett. 107, 181802 (2011) arXiv:1108.0015 [hep-ex]].

[6] D. V. Forero, M. Tortola and J. W. F. Valle, Phys. Rev. D 86, 073012 (2012) arXiv:1205.4018 [hep-ph]].

[7] G. L. Fogli, E. Lisi, A. Marrone, D. Montanino, A. Palazzo and A. M. Rotunno, Phys. Rev. D 86, 013012 (2012) arXiv:1205.5254 [hep-ph]].

[8] M. C. Gonzalez-Garcia, M. Maltoni, J. Salvado and T. Schwetz, arXiv:1209.3023 [hepph].

[9] P. F. Harrison, D. H. Perkins and W. G. Scott, Phys. Lett. B 530 (2002) 167 hep-ph/0202074]; P. F. Harrison and W. G. Scott, Phys. Lett. B 535 (2002) 163 
hep-ph/0203209]; Z. -z. Xing, Phys. Lett. B 533 (2002) 85 hep-ph/0204049 ; X. G. He and A. Zee, Phys. Lett. B 560, 87 (2003) hep-ph/0301092.

[10] H. -J. He and F. -R. Yin, Phys. Rev. D 84, 033009 (2011) arXiv:1104.2654 [hepph]]; Y. Shimizu, M. Tanimoto and A. Watanabe, Prog. Theor. Phys. 126, 81 (2011) arXiv:1105.2929 [hep-ph]]; J. -M. Chen, B. Wang and X. -Q. Li, Phys. Rev. D 84, 073002 (2011) arXiv:1106.3133 [hep-ph]]; Z. -z. Xing, arXiv:1106.3244 [hep-ph]]; E. Ma and D. Wegman, Phys. Rev. Lett. 107, 061803 (2011) [arXiv:1106.4269 [hep-ph]]; Y. j. Zheng and B. -Q. Ma, arXiv:1106.4040 [hep-ph]]; X. -G. He and A. Zee, Phys. Rev. D 84, 053004 (2011) arXiv:1106.4359 [hep-ph]]; S. Zhou, Phys. Lett. B 704, 291 (2011) [arXiv:1106.4808 [hep-ph]]; N. Haba and R. Takahashi, Phys. Lett. B 702, 388 (2011) [arXiv:1106.5926 [hep-ph]]; D. Meloni, JHEP 1110, 010 (2011) arXiv:1107.0221 [hep-ph]]; S. Morisi, K. M. Patel and E. Peinado, Phys. Rev. D 84, 053002 (2011) arXiv:1107.0696 [hep-ph]]; W. Chao and Y. -j. Zheng, arXiv:1107.0738 [hep-ph]; H. Zhang and S. Zhou, Phys. Lett. B 704, 296 (2011) [arXiv:1107.1097 [hep-ph]]; X. Chu, M. Dhen and T. Hambye, JHEP 1111, 106 (2011) arXiv:1107.1589 [hep-ph]]; P. S. Bhupal Dev, R. N. Mohapatra and M. Severson, Phys. Rev. D 84, 053005 (2011) [arXiv:1107.2378 [hep-ph]]; S. Antusch and V. Maurer, Phys. Rev. D 84, 117301 (2011) [arXiv:1107.3728 [hep-ph]]; W. Rodejohann, H. Zhang and S. Zhou, Nucl. Phys. B 855, 592 (2012) arXiv:1107.3970 [hep-ph]]; Y. H. Ahn, H. -Y. Cheng and S. Oh, arXiv:1107.4549 [hep-ph]]; S. F. King and C. Luhn, JHEP 1109, 042 (2011) [arXiv:1107.5332 [hep-ph]]; Q. -H. Cao, S. Khalil, E. Ma and H. Okada, Phys. Rev. D 84, 071302 (2011) [arXiv:1108.0570 [hep-ph]]; D. Marzocca, S. T. Petcov, A. Romanino and M. Spinrath, JHEP 1111, 009 (2011) arXiv:1108.0614 [hep-ph]]; S. -F. Ge, D. A. Dicus and W. W. Repko, arXiv:1108.0964 [hep-ph]]; Riazuddin, arXiv:1108.1469 [hep-ph]; F. Bazzocchi, arXiv:1108.2497 [hep-ph]]; T. Araki and C. -Q. Geng, JHEP 1109, 139 (2011) [arXiv:1108.3175 [hep-ph]]; S. Antusch, S. F. King, C. Luhn and M. Spinrath, Nucl. Phys. B 856, 328 (2012) [arXiv:1108.4278 [hep-ph]]; H. Fritzsch, Z. -z. Xing and S. Zhou, JHEP 1109, 083 (2011) arXiv:1108.4534 [hep-ph]]; R. N. Mohapatra and M. K. Parida, Phys. Rev. D 84, 095021 (2011) arXiv:1109.2188 [hep-ph]]; A. Rashed and A. Datta, arXiv:1109.2320 [hep-ph]]; P. O. Ludl, S. Morisi and E. Peinado, arXiv:1109.3393 [hep-ph]]; N. Okada and Q. Shafi, arXiv:1109.4963 [hepph]; A. Aranda, C. Bonilla and A. D. Rojas, arXiv:1110.1182 [hep-ph]]; G. -J. Ding, L. L. Everett and A. J. Stuart, Nucl. Phys. B 857, 219 (2012) arXiv:1110.1688 [hep-ph]]; D. Meloni, arXiv:1110.5210 [hep-ph]; S. Dev, S. Gupta, R. R. Gautam and L. Singh, Phys. Lett. B 706, 168 (2011) arXiv:1111.1300 [hep-ph]]; K. N. Deepthi, S. Gollu and R. Mohanta, arXiv:1111.2781 [hep-ph]; A. Rashed, arXiv:1111.3072 [hep-ph]; W. Buchmuller, V. Domcke and K. Schmitz, arXiv:1111.3872 [hep-ph]; I. d. M. Varzielas, arXiv:1111.3952 [hep-ph]; R. de Adelhart Toorop, F. Feruglio and C. Hagedorn, arXiv:1112.1340 [hepph]]; S. F. King and C. Luhn, arXiv:1112.1959 [hep-ph]; S. Gupta, A. S. Joshipura and K. M. Patel, arXiv:1112.6113 [hep-ph]. H. Ishimori and T. Kobayashi, Phys. Rev. 
D 85, 125004 (2012) arXiv:1201.3429 [hep-ph]]; S. Dev, R. R. Gautam and L. Singh, Phys. Lett. B 708, 284 (2012) [arXiv:1201.3755 [hep-ph]]. P. S. Bhupal Dev, B. Dutta, R. N. Mohapatra and M. Severson, Phys. Rev. D 86, 035002 (2012) arXiv:1202.4012 [hep-ph]]; X. Zhang and B. -Q. Ma, Phys. Lett. B 710, 630 (2012) arXiv:1202.4258 [hepph]]; I. K. Cooper, S. F. King and C. Luhn, JHEP 1206, 130 (2012) arXiv:1203.1324 [hep-ph]]; Z. -Z. Xing, Chin. Phys. C 36, 281 (2012) [arXiv:1203.1672 [hep-ph]]; Y. L. Wu, Phys. Lett. B 714, 286 (2012) [arXiv:1203.2382 [hep-ph]]; Y. BenTov and A. Zee, Phys. Lett. B 714, 80 (2012) [arXiv:1203.2671 [hep-ph]]; H. -J. He and X. J. Xu, arXiv:1203.2908 [hep-ph]; D. Meloni, JHEP 1205, 124 (2012) arXiv:1203.3126 [hep-ph]]. Y. H. Ahn and S. K. Kang, arXiv:1203.4185 [hep-ph]; I. d. M. Varzielas and G. G. Ross, arXiv:1203.6636 [hep-ph]; D. Hernandez and A. Y. .Smirnov, arXiv:1204.0445 [hep-ph]; C. Hagedorn and D. Meloni, Nucl. Phys. B 862, 691 (2012) arXiv:1204.0715 [hep-ph]]; M. Fukugita, Y. Shimizu, M. Tanimoto and T. T. Yanagida, Phys. Lett. B 716, 294 (2012) [arXiv:1204.2389 [hep-ph]]; S. Zhou, arXiv:1205.0761 [hep-ph]; C. Hagedorn, S. F. King and C. Luhn, arXiv:1205.3114 [hep-ph]; G. Altarelli, F. Feruglio, L. Merlo and E. Stamou, JHEP 1208, 021 (2012) arXiv:1205.4670 [hep-ph]]; G. Altarelli, F. Feruglio and L. Merlo, arXiv:1205.5133 [hep-ph]; A. Meroni, S. T. Petcov and M. Spinrath, arXiv:1205.5241 [hep-ph]; M. J. Baker, J. Bordes, H. M. Chan and S. T. Tsou, arXiv:1206.0199 [hep-ph]; X. Zhang and B. -Q. Ma, arXiv:1206.0519 [hep-ph]; E. Ma, A. Natale and A. Rashed, Int. J. Mod. Phys. A 27, 1250134 (2012) arXiv:1206.1570 [hepph]]; N. Haba and R. Takahashi, arXiv:1206.2793 [hep-ph]; A. G. Dias, A. C. B. Machado and C. C. Nishi, arXiv:1206.6362 [hep-ph]; P. M. Ferreira, W. Grimus, L. Lavoura and P. O. Ludl, arXiv:1206.7072 [hep-ph]; G. Altarelli, F. Feruglio, I. Masina and L. Merlo, arXiv:1207.0587 [hep-ph]; Y. BenTov, X. -G. He and A. Zee, arXiv:1208.1062 [hepph]; W. Rodejohann and H. Zhang, arXiv:1207.1225 [hep-ph]; R. N. Mohapatra and C. C. Nishi, arXiv:1208.2875 [hep-ph].

[11] R. d. A. Toorop, F. Feruglio and C. Hagedorn, Phys. Lett. B 703, 447 (2011) arXiv:1107.3486 [hep-ph]].

[12] G. -J. Ding, Nucl. Phys. B 862, 1 (2012) arXiv:1201.3279[hep-ph]].

[13] S. F. King, C. Luhn and A. J. Stuart, arXiv:1207.5741 [hep-ph].

[14] S. M. Boucenna, S. Morisi, M. Tortola and J. W. F. Valle, Phys. Rev. D 86, 051301 (2012) arXiv:1206.2555 [hep-ph]].

[15] S. F. King, arXiv:1205.0506 [hep-ph]; S. Antusch, C. Gross, V. Maurer and C. Sluka, arXiv:1205.1051 [hep-ph];

[16] H. Minakata and A. Y. .Smirnov, Phys. Rev. D 70, 073009 (2004) hep-ph/0405088; N. Li and B. -Q. Ma, Eur. Phys. J. C 42, 17 (2005) hep-ph/0504161]; N. Qin and B. Q. Ma, 
Phys. Lett. B 702, 143 (2011) arXiv:1106.3284 [hep-ph]]; Y. H. Ahn, H. -Y. Cheng and S. Oh, Phys. Rev. D 83, 076012 (2011) arXiv:1102.0879 [hep-ph]].

[17] C. D. Froggatt and H. B. Nielsen, Nucl. Phys. B 147 (1979) 277.

[18] M. Leurer, Y. Nir and N. Seiberg, Nucl. Phys. B 398, 319 (1993) hep-ph/9212278; Nucl. Phys. B 420, 468 (1994) hep-ph/9310320.

[19] E. Dudas, C. Grojean, S. Pokorski and C. A. Savoy, Nucl. Phys. B 481, 85 (1996) hep-ph/9606383];K. Choi, E. J. Chun and H. D. Kim, Phys. Lett. B 394, 89 (1997) hep-ph/9611293]; N. Irges, S. Lavignac and P. Ramond, Phys. Rev. D 58, 035003 (1998) hep-ph/9802334; J. K. Elwood, N. Irges and P. Ramond, Phys. Rev. Lett. 81, 5064 (1998) hep-ph/9807228; J. R. Ellis, G. K. Leontaris and J. Rizos, JHEP 0005, 001 (2000) hep-ph/0002263]; H. K. Dreiner and M. Thormeier, Phys. Rev. D 69, 053002 (2004) [hep-ph/0305270]; H. K. Dreiner, H. Murayama and M. Thormeier, Nucl. Phys. B 729, 278 (2005) hep-ph/0312012]; M. -C. Chen, D. R. T. Jones, A. Rajaraman and H. -B. Yu, Phys. Rev. D 78, 015019 (2008) [arXiv:0801.0248 [hep-ph]]; L. F. Duque, D. A. Gutierrez, E. Nardi and J. Norena, Phys. Rev. D 78, 035003 (2008) [arXiv:0804.2865 [hep-ph]].

[20] G. Altarelli, F. Feruglio and L. Merlo, JHEP 0905, 020 (2009) arXiv:0903.1940 [hep$\mathrm{ph}]$.

[21] L. Wolfenstein, Phys. Rev. Lett. 51, 1945 (1983).

[22] J. Schechter and J. W. F. Valle, Phys. Rev. D 22 (1980) 2227; W. Rodejohann and J. W. F. Valle, Phys. Rev. D 84, 073011 (2011) [arXiv:1108.3484 [hep-ph]].

[23] H. Fusaoka and Y. Koide, Phys. Rev. D 57, 3986 (1998) hep-ph/9712201; G. Ross and M. Serna, Phys. Lett. B 664, 97 (2008) [arXiv:0704.1248 [hep-ph]]; Z. -z. Xing, H. Zhang and S. Zhou, Phys. Rev. D 77, 113016 (2008) arXiv:0712.1419 [hep-ph]].

[24] A. Rasin and J. P. Silva, Phys. Rev. D 49, 20 (1994) hep-ph/9309240.

[25] Y. Grossman and Y. Nir, Nucl. Phys. B 448, 30 (1995) hep-ph/9502418; Y. Grossman, Y. Nir and Y. Shadmi, JHEP 9810, 007 (1998) hep-ph/9808355; Y. Nir and Y. Shadmi, JHEP 9905, 023 (1999) hep-ph/9902293.

[26] L. J. Hall and A. Rasin, Phys. Lett. B 315, 164 (1993), hep-ph/9303303.

[27] M. B. Green and J. H. Schwarz, Phys. Lett. B 149, 117 (1984).

[28] L. E. Ibanez and G. G. Ross, Phys. Lett. B 332, 100 (1994) hep-ph/9403338; P. Binetruy and P. Ramond, Phys. Lett. B 350, 49 (1995) hep-ph/9412385]; V. Jain and R. Shrock, Phys. Lett. B 352, 83 (1995) hep-ph/9412367]; E. Dudas, S. Pokorski and C. A. Savoy, Phys. Lett. B 356, 45 (1995) hep-ph/9504292; P. Binetruy, S. Lavignac and 
P. Ramond, Nucl. Phys. B 477, 353 (1996) hep-ph/9601243]; P. H. Chankowski, K. Kowalska, S. Lavignac and S. Pokorski, Phys. Rev. D 71, 055004 (2005) hep-ph/0501071.

[29] A. E. Nelson and D. Wright, Phys. Rev. D 56, 1598 (1997) [hep-ph/9702359].

[30] H. Georgi and C. Jarlskog, Phys. Lett. B 86, 297 (1979).

[31] J. R. Ellis and M. K. Gaillard, Phys. Lett. B 88, 315 (1979).

[32] G. Altarelli, F. Feruglio and I. Masina, JHEP 0011, 040 (2000) hep-ph/0007254.

[33] J. Schechter and J. W. F. Valle, Phys. Rev. D 25, 2951 (1982).

[34] A. Osipowicz et al. [KATRIN Collaboration], arXiv:hep-ex/0109033; see also: http://www-ik.fzk.de/ katrin/index.html

[35] C. Arnaboldi et al. [CUORE Collaboration], Nucl. Instrum. Meth. A 518, 775 (2004) hep-ex/0212053; R. Ardito, C. Arnaboldi, D. R. Artusa, F. T. Avignone, III, M. Balata, I. Bandac, M. Barucci and J. W. Beeman et al., hep-ex/0501010.

[36] S. R. Elliott [MAJORANA Collaboration], J. Phys. Conf. Ser. 173, 012007 (2009) arXiv:0807.1741 [nucl-ex]]; V. E. Guiseppe et al. [Majorana Collaboration], IEEE Nucl. Sci. Symp. Conf. Rec. 2008, 1793 (2008) [arXiv:0811.2446 [nucl-ex]].

[37] I. Abt, M. F. Altmann, A. Bakalyarov, I. Barabanov, C. Bauer, E. Bellotti, S. T. Belyaev and L. B. Bezrukov et al., hep-ex/0404039; S. Schonert (GERDA Collaboration), J. Phys. Conf. Ser. 203, 012014 (2010).

[38] M. Cannoni, J. Ellis, M. E. Gomez and S. Lola, arXiv:1301.6002 [hep-ph].

[39] L. Zhan, Y. Wang, J. Cao and L. Wen, Phys. Rev. D 79, 073007 (2009) arXiv:0901.2976 [hep-ex]]; X. Qian, D. A. Dwyer, R. D. McKeown, P. Vogel, W. Wang, and C. Zhang, arXiv:1208.1551 [hep-ex].

[40] P. Binetruy, S. Lavignac, S. T. Petcov and P. Ramond, Nucl. Phys. B 496, 3 (1997) hep-ph/9610481; S. Lola and G. G. Ross, Nucl. Phys. B 553, 81 (1999) hep-ph/9902283; M. Tanimoto, Phys. Lett. B 456, 220 (1999) hep-ph/9901210]; M. S. Berger and K. Siyeon, Phys. Rev. D 62, 033004 (2000) hep-ph/0003121; M. S. Berger and K. Siyeon, Phys. Rev. D 64, 053006 (2001) hep-ph/0005249]; J. Sato and K. Tobe, Phys. Rev. D 63, 116010 (2001) hep-ph/0012333.

[41] Y. Fukuda et al. [Super-Kamiokande Collaboration], Phys. Rev. Lett. 81, 1562 (1998) hep-ex/9807003.

[42] M. Maltoni, T. Schwetz, M. A. Tortola and J. W. F. Valle, New J. Phys. 6, 122 (2004) hep-ph/0405172. 